pylorus in infants. The flattening of the mucosa can hardly be considered as an evidence of permanent closure. The cystic dilatations of the glands are probably mechanical in origin, though a few similar cysts have been seen in the mucosa of the normal pylorus of infants. In the case of an infant a few weeks old, operated upon for pyloric stenosis and which died a few days later, only slightly dilated pyloric glands were found. This fact supports the belief that in the case here reported, the long-continued closure of the pylorus was the cause of the glandular dilatation.

This is the first time, so far as we know, that any one has had the opportunity of studying the anatomical conditions in a case of infantile pyloric stenosis at so long a time as six and one-half months after a successful gastro-enterostomy. This case proves that in some instances, at least, the stenosis is not due to spasm, that medical treatment cannot be of any avail, and that the only hope for relief and life lies in surgical intervention. It also proves that an infant can live and thrive for many months, although all the food passes through the gastro-enterostomy opening and none through the pylorus. It suggests that the condition of organic infantile pyloric stenosis is a permanent one, that there is no tendency toward a restoration of normal conditions, and that there is little hope of the pylorus ever resuming its functions. It is not safe to draw conclusions from these suggestions, however, as it is possible that with the lapse of time and the growth of the parts, changes may occur which will result in the restoration of normal conditions and function. These points can only be decided by the actual observation of the anatomical conditions in cases dead at longer intervals after successful operations.

\section{A CASE OF CONGENITAL STENOSIS OF THE PYLORUS.*}

BY CHARLES W. TOWNSEND, M.D., BOSTON.

A MALE infant, sixteen days old, was admitted to the Boston Floating Hospital on Aug. 9, 1907, and was seen by me at the beginning of my service, four days later. The infant was born at full term on July 24, and had been breast fed until its admission to the hospital. Since then it had had its stomach washed, it had been fed on sugar water, whey and breast milk. Soon after birth regurgitation occurred, following each nursing. Later the vomiting became a marked symptom, generally after each feeding, and sometimes explosive or projectile in character. If the food were retained for one or two feedings, the total amount would afterwards be vomited. Almost continuous regurgitation of food was sometimes noticed, so that the napkin under the head was always wet.

The dejections were black and stringy, and their appearance, even on the twentieth day of the child's life, when I began my service, was that of meconium. Apparently no food found its way through the intestine, yet the infant appeared in fairly good condition, and showed but little loss of flesh or color.

The examination of the abdomen showed slightly greater prominence in the upper than in the lower * Read before the Boston Society for Medical Improvement, Jan.
27, 1908. portion, and an increase in the area of stomachic resonance. At no time could any mass be felt at the pylorus, and no peristaltic movements could be seen.

The apparently good condition of the infant made it seem probable that some food was digested, and passed through the intestinal canal, but careful observation showed that as much was vomited as was received, and at no time was there any evidence of food material in the stools.

The case was referred to'Dr. James S. Stone, but as the infant gained an ounce, and as the results of examination of the abdomen were so nega tive it seemed advisable to postpone the operation until the 24th day of the infant's life on Aug. 17.

OPERATION BYY DR. J. S. STONE.

Ether anesthesia. Through a perpendicular incision through the outer border of the left rectus the stomach was exposed and was found to be much dilated. The pylorus was felt to be firm and enlarged. The whole large and small intestine was found to be collapsed. The lower anterior part of the greater curvature of the stomach was seized with clamps and a loop of small intestine immediately below the duodenum withdrawn and seized with clamps. An anastomosis was then made with a double row of silk sutures. During the operation the child stopped breathing and artificial respiration had to be continued for several minutes. Aside from this the greatest diffculty was in determining which loop of the collapsed small intestine led up to the duodenum. It seemed wiser in view of the condition of the child to do an anterior rather than a posterior gastro-enterostomy because the latter operation would have added a little to the difficulties. The child stood the operation, which was prolonged by the delay mentioned, fairly well and rallied well afterward. The abdominal wound was closed without drainage.

The operation was well borne by the infant. On the second day diluted mother's milk was given, and about half was retained. The movements for the first time in the infant's life showed traces of food. Vomiting continued, however, from time to time, the infant grew weaker and on the third day after operation, the twenty-seventh day of its life, it died.

The interesting points in the case are the facts that so little weight was lost, and that the color and general condition of the infant remained so good, notwithstanding the occlusion of the pylorus. The birth weight is not known. The weight on the 17 th day was $7 \mathrm{lb} .5 \frac{1}{2} \mathrm{oz}$; on the 19 th day, $7 \mathrm{lb} . \frac{1}{2} \mathrm{oz}$; on the $21 \mathrm{st}$ day, $6 \mathrm{lb} .11 \frac{1}{2} \mathrm{oz}$; ; on the $22 \mathrm{~d}$ day, $6 \mathrm{lb}$. $12 \frac{1}{2} \mathrm{oz}$; on the 24th day, $6 \mathrm{lb}$. $11 \mathrm{oz}$.

Autopsy by Dr. S. B. Wolbach, on Aug. 20, 1907 :

Body of a well-formed, fairly well-nourished, white male infant. ... On the anterior surface of the stomach, close to the greater curvature, and about $6 \mathrm{~cm}$. from the pylorus, there is sutured a loop of the small intestine. . . . The union between small intestine and stomach is firm and dry. There are no adhesions to other structures. The pylorus is felt as a very dense cylindrical mass about $1 \mathrm{~cm}$. long and $\frac{3}{4} \mathrm{~cm}$. in diameter. ... Pylorus is tightly contracted; with slight pressure a tapering probe dilates the opening to $2 \mathrm{~cm}$. in circumference. On section there is an enormous thickening of the muscle layer, forming a well-defined band en- 
circling the pylorus, $1 \mathrm{~cm}$. in width and about $2 \mathrm{~mm}$. in thickness. The mucous membrane enclosed by the pyloric ring lies in longitudinal folds. There is no evidence of scar tissue. The stomach contains a small quantity of clear liquid with white flocculi. The distended loop of the duodenum contains brownish, tenaceous material, which is inoffensive. The collapsed small intestine is absolutely empty, except for an occasional small mucous particle. The colon is normal. The lower portion contains a little thin, .yellowish inoffensive material.

Anatomical diagnosis : Congenital hypertrophy of the pylorus. Gastro-enterostomy operation wound.

\section{THE SCOPE OF THERAPEUTIC INOCULATION.*}

BY G. P. SANBORN, M.D., BOSTON,

Formerly Assistant in Bacteriology, Harvard Medical School ; formerly Assistant to Dr. John H. McCollom, South Department, Boston City Hospital ; formerly A ssistant in Prof.
Laboratory, St. Mary's Hospital, London.

The illuminating lecture that Prof. Sir Almroth E. Wright delivered in Boston, somewhat over a year ago, profoundly interested me. It seemed to offer us some hope of being enabled to treat successfully certain infections, over which we have hitherto been practically powerless. I had particularly in mind the septic complications of diphtheria and of the exanthemata, which are responsible for so great a proportion of the mortality in those particular diseases. The success of anti-toxin in diphtheria which I have seen during a two years' service at the South Department of this hospital served by its very contrast to emphasize the lack of specific treatment in other infections. It was my privilege last June to go to London for the purpose of study under Sir Almroth Wright. I entered his laboratory as a beginner, never having carried out, or seen carried out, either the opsonic laboratory technic or the methods of treatment, and I therefore can say that I was absolutely unprejudiced either for or against Wright's particular methods.

After less than three weeks spent in acquiring a rudimentary knowledge of the technic, I grasped an opportunity that offered to spend the month of July in the study of the general subject of immunity at the University of Cambridge under Prof. Sims Woodhead, Dr. Louis Cobbett and others. When I returned to Wright's laboratory Aug. 1, I felt myself much better equipped to take up the study of his methods and to judge for myself as to their usefulness, because not only had I reviewed the subject of immunity, but had acquired the view points of the very able men above mentioned, as applied to opsonic methods. Although Wright's work was discussed absolutely fairly, I could see that there was little enthusiasm at Cambridge over opsonic methods or the results of treatment by bacterial vaccine. This I found emanated from the fact that certain investigators there had completed a careful and exhaustive study into the accuracy of Wright's method. Their results showed that the possible error might be at any time from $25 \%$ to $100 \%$ or more, and they are

* Read at a clinical meeting at the Boston City Hospital, March 26,1908 . quite in accord with the results of many other investigators based on a much less elaborate study. I was therefore in a more or less skeptical frame of mind when I returned to Wright's laboratory but I still felt that there was to be balanced against their unfavorable evidence, the result of a few months' work, the careful painstaking investigations of Wright and Douglas and their co-workers, extending over several years, to say nothing of that of Bullock, the whole, the result of carefully directed effort along one special line.

It happened that, at the time of my return, Wright was in need of an assistant for routine work and was good enough to appoint me for this position which I held for five months. I was thus enabled by engaging in the daily routine to become familiar with opsonic technic and, by being present at the clinics, to carefully follow the cases in their treatment and progress. The result of my work there, which I consider first in importance, was that I was enabled to follow certain investigations that were being carried out regarding the reasons for inaccuracy in opsonic determination; that is, the sources of error in carrying out the technic. These researehes have not yet been published, and I have yet to see evidence that they are recognized by any of the important critics of Wright and his methods. These researches will in due time be published from Wright's laboratory, and although I have a record of the experiments and results, it is, of course, not for me to give them in detail. I feel permitted, however, to say that one who is not cognizant of these sources of error cannot hope to obtain accurate opsonic indices unless he chances to be lucky. Given the most perfect technic that any one can develop after three months' constant practice, there still remain four or five at present unrecognized sources of error which, unless guarded against, may produce inaccuracies varying from $25 \%$ to $200 \%$ or $300 \%$, and I have no doubt that some of them are responsible for the very bad results that have led many workers to give up opsonic determination. With a realization of these sources of error and of methods to guard against them, the limits of variation in the opsonic indices of normal people, according to a multitude of evidence that I have obtained from the records of Wright's laboratory, should be but $10 \%$ in three quarters of normal cases examined and not more than $20 \%$ in the remaining one quarter. There is, I submit, no reason to anticipate a greater range of variation due to errors of technic in the indices of patients than in those of normal people. I can say from experience, that a possible error of $10 \%$ or occasionally of $20 \%$ I know to be consistent with usefulness for the opsonic index.

During my first two months of service, I put the opsonic index to as many tests as possible and was soon able to convince myself that, as done in Wright's laboratory, it is sufficiently accurate to be useful. I was able to see it applied and to apply it successfully in the determination of the maximum dose of tuberculin at the earliest possible moment and of the proper interval between 